\title{
Dimitri Karadimas et le paradigme entomologiste
}

\section{Emmanuel Désveaux}

\section{(2) OpenEdition}

Journals

Édition électronique

URL : https://journals.openedition.org/jsa/15879

DOI : $10.4000 /$ jsa. 15879

ISSN : $1957-7842$

Éditeur

Société des américanistes

Édition imprimée

Date de publication : 15 juin 2018

Pagination : 251-254

ISSN : 0037-9174

\section{Référence électronique}

Emmanuel Désveaux, « Dimitri Karadimas et le paradigme entomologiste », Journal de la Société des américanistes [En ligne], 104-1 | 2018, mis en ligne le 15 juin 2018, consulté le 04 septembre 2022 URL : http://journals.openedition.org/jsa/15879; DOI : https://doi.org/10.4000/jsa.15879 


\title{
Dimitri Karadimas et le paradigme entomologiste
}

\author{
Emmanuel DésveAuX *
}

Je connaissais Dimitri Karadimas de longue date puisqu'il faisait partie de la poignée d'étudiants, quatre ou cinq tout au plus, qui composait le public du cours intitulé « Lire les Mythologiques » dont j'eus brièvement la charge à l'université de Nanterre, tout de suite après avoir passé mon doctorat. Tout cela remonte au milieu des années 1980. Bien sûr, nous nous sommes un peu perdus de vue, notamment parce qu'il se rendait souvent sur son terrain au début de sa carrière d'ethnologue. Plus tard, à d'autres moments de nos existences respectives, nous avons eu l'occasion de nous croiser de nouveau - il entra au Laboratoire d'anthropologie sociale au moment où je le quittai - et de prendre plaisir à échanger, aussi librement que faire se peut. À défaut de lier une véritable amitié, état devenu de plus en plus rare dans notre monde professionnel, nous avons toujours gardé, je crois, de l'estime l'un pour l'autre. Une sorte de confiance mutuelle à moyenne distance, pourrait-on dire. Mais il faut bien reconnaître que durant toutes ces années, je ne suivais pas régulièrement la production éditoriale de Dimitri Karadimas. Avoir été nommé pré-rapporteur de son dossier d'habilitation par mes collègues de l'École des hautes études en sciences sociales à l'automne 2015 me fit découvrir alors l'immensité de son travail. Ce fut une révélation, compte tenu de son ampleur (voir sa bibliographie) mais surtout de sa richesse et de sa fécondité. Dimitri Karadimas portait en effet un projet d'une forte ambition théorique sous l'étendard d'une notion de prime abord assez absconse, la paréidolie (image de la proie). Il s'agit en réalité de tenir ensemble les deux pôles les plus extrêmes de toute démarche anthropologique, à savoir le pôle psychologique et le pôle diachronique et ce, sans sombrer dans un évolutionnisme primaire. L'invocation de la psychologie n'est pas pure coquetterie ici puisque, disait Claude Lévi-Strauss, l'anthropologie n'est jamais en définitive qu'une psychologie. Mais nous pourrions tout aussi bien parler de pôle cognitiviste, afin de moderniser le propos.

\footnotetext{
* École des hautes études en sciences sociales [emmanuel.desveaux@ehess.fr].
} 
L'origine de l'anthropologie de Dimitri Karadimas tient à son expérience ethnographique des Miraña, une population du Nord-Ouest amazonien. Dans ses premiers travaux, il insistait sur le corps humain comme référent quasi-exclusif d'un très grand nombre de représentations, voire de toutes les représentations. Il reprenait l'argument mais en lui donnant une direction inattendue. Dans l'ouvrage inédit qui constituait le cœur de son dossier d'habilitation, Dieux, guerriers, parasites célestes. Perception, mythes et images en Amérique du $S u d$, Karadimas se penchait sur la question iconologique. Il envisageait d'abord une vaste aire culturelle qui englobe l'ensemble des populations de l'Amazonie occidentale - il s'agit en gros de l'aire où prévaut le fameux rituel Yurupari - et les zones andines adjacentes. Cela est de bonne méthode car l'échelle considérée permet un vrai comparatisme, et ce d'autant plus qu'elle permet d'introduire une dimension diachronique, l'essentiel de l'iconologie des hautes terres étant révélé par l'archéologie. Il s'agit donc pour lui de se livrer à une large comparaison du matériel iconographique provenant de toute la zone étudiée mettant pratiquement sur le même plan l'ethnographie contemporaine, l'ethnographie historique - celle datant du siècle dernier et en particulier celle, très riche, de Koch-Grünberg, mais aussi celles de Reichel-Dolmatoff et de Hugh-Jones - et, enfin, l'archéologie. L'iconographie des masques miraña est ainsi susceptible d'être immédiatement rapprochée de celle figurée sur les céramiques et les tissus anciens, tandis que des objets « classiques » de l'ethnographie régionale, telles les trompes du Yurupari, prennent une signification plus riche que toutes les interprétations qui qui en avaient été données jusqu'alors.

Karadimas a fait ici une découverte, à nos yeux, majeure : en réalité, la référence ultime de toute l'iconographie considérée ne réside pas dans le corps humain ainsi qu'il le voyait lui-même dans un premier temps, pas plus que la faune ordinaire - j'entends par là celle qui appartient à la même échelle phénoménologique que l'humain, c'est-à-dire, les aigles, les jaguars, les serpents ainsi que cela est affirmé en règle générale. Non, la référence est pour l'essentiel entomologiste. Ce sont les araignées, les guêpes parasitoïdes, les Eucharitidae, les hyménoptères, les chenilles qui mènent désormais le bal de l'iconologie régionale. Ce ne sont pas seulement les insectes que la sensibilité immédiate autochtone aurait retenus comme pertinente dans un regard microscopique généralisé sur le monde, ce sont de préférence les insectes prédateurs, ceux qui précisément pondent leurs œufs dans le corps, encore vivant, de leur proie. Ainsi les guêpes pompiles, prédatrices exclusives des araignées, s'attaquent à leur victime, les amputent des pattes afin de mieux pouvoir les déplacer et les installer à leur aise là où elles pourront en faire un nid confortable. Leurs larves se nourriront directement des sucs qu'exhale le corps " encore chaud " de la pauvre mygale. Le grand intérêt de la découverte de Karadimas tient à son fort rendement en termes d'élucidation de formes iconographiques. Une 
bonne partie du corpus iconographique est ainsi réinterprétée. Les « cornes » du diabolo n'en sont plus ; il s'agit désormais de mandibules. Les yeux parfaitement circulaires renvoient à ceux des insectes, les « cornes 》 - ou les crocs - à des mandibules; les émanations latérales d'un corps ne sont plus des marques de rayonnement des personnages mais des représentations des fines pattes latérales des insectes. Les êtres hybrides n'en sont pas vraiment : ils ne sont que l'insecte associé à sa victime, chenille ou araignée, qu'il transporte ou avec lequel il « s'accouple», en fait y dépose ses œufs. Rien, aucune figure, aucun dessin ne semble pouvoir résister à cette relecture entomologique. Personnellement, elle me convainc largement.

Ce faisant, la démonstration que livre Karadimas nous intéresse puisqu'elle accrédite son explication des notions amérindiennes de la conception dans la mesure où la forme insecte parasitoïde et le corps réceptacle offrent une image de l'accouplement sexuel. Bien qu'elle inclue éventuellement la notion de contamination, cette option phénoménologique exclut l'idée même d'hérédité biologique. C'est exactement ce que j'ai tenté de montrer dans mes différents travaux depuis plusieurs années déjà. Car ce qui distingue les humains des insectes, outre la différence d'échelle, c'est le langage, de même que ce qui distingue les lombrics des trompes du Yurupari, c'est qu'elles «parlent »... là où les vers de terre restent irrémédiablement muets.

Ce paradigme entomologiste que Karadimas dévoile pour l'aire culturelle qu'il embrasse - l'Amazonie occidentale et les Andes - suscite des interrogations chez l'américaniste. Vaut-il uniquement pour cette région ou pour l'ensemble du Nouveau Monde ? Et si ce n'est pas le cas, pourquoi se serait-il cantonné à cette zone ? Il est en tout cas troublant pour moi de le voir ressurgir en Australie, ainsi que je l'ai montré dans mon livre Avant le genre, triptyque d'anthropologie hardcore, mais il est vrai, avec pour arrière-plan, une conception tout à fait différente de la relation entre les sexes.

Dans son mémoire de synthèse pour obtenir l'habilitation à diriger des recherches, après le passage du corps humain au miroir de l'insecte, Karadimas franchit une troisième étape dans sa quête en s'intéressant à ce que l'on décrit en général comme des êtres chimériques en revenant en quelque sorte à son anthropomorphisme initial. Ces entités apparaissent en effet comme autant de déclinaisons d'un anthropomorphisme physiologique généralisé, permettant de transposer les attributs d'une espèce vivante à une autre, ou plutôt de convertir une partie de corps d'une espèce en celle d'une autre espèce. La pince du crabe se superpose à la main du malade, au moins telle qu'elle est perçue par les esprits convoqués par le chaman afin de la soigner. Ici, s'immisce le perspectivisme de Viveiros de Castro, relayé par la théorie d'origine cognitiviste de « saillances ». Dimitri Karadimas revient alors sur la question des classifications zoologiques, en montrant qu'en définitive le principe de monstruosité sert de soubassement à tout processus d'identification fine. Le monstre ne se situe pas à la marge du 
système des classifications, tel un validateur, comme chez Sperber, mais en son cœur même. Je cite :

L'identification d'une espèce et non d'un genre procède par l'ajustement, au sein d'une même catégorie d'existants, de caractères spécifiques qui permettent de discriminer une espèce par rapport à l'ensemble des autres. Or ce caractère spécifique emprunte le plus souvent $[. .$.$] le nom d'une autre espèce... pour le$ combiner et, de la sorte, marquer l'espèce. Les noms composés sont ainsi emblématiques de ce processus à l'interface entre perception et identification. Dans ces espèces, un caractère anatomique particulier renvoie à un autre domaine que celui de l'espèce-source.

Ainsi par exemple, le poisson-chat ou le requin-marteau. On comprend mieux maintenant la signification de la paréidolie : le processus perceptif qui conduit à assoir une identification stable a recours à une analogie avec une autre représentation appartenant à une autre espèce animale, mais ayant en quelque sorte une extension préalable plus large. C'est par ce biais que Dimitri Karadimas, non seulement remet en cause l'arbitraire du signe saussurien et instruit à nouveaux frais l'idée d'une motivation du signe, serait-elle seconde, mais « ose » en quelque sorte renouer avec la dimension diachronique.

Karadimas construit une œuvre très originale qui s'enracine, bien sûr, dans celle de Lévi-Strauss (quel américaniste pourrait l'ignorer ?). Sa façon en est plutôt contrapuntique puisqu'elle réfute en partie la notion de transformation logique par inversion pour s'inscrire dans le sillage du néo-lévy-bruhlisme de Philippe Descola qui a le mérite de faciliter le dialogue entre anthropologie et sciences cognitives.

J'ajoute un point sur la méthode de Dimitri Karadimas. Son travail repose largement sur 1'usage des nouvelles techniques de documentation. Il a su tirer profit du gisement d'images que représente désormais l'Internet, que ce soit pour établir des savoirs objectifs en matière d'entomologie ou pour récoler de la façon la plus exhaustive possible des données sur la céramique précolombienne à partir des banques qui leur sont dédiées. Si un tel travail semblait encore inimaginable il y a une vingtaine d'années, il faut reconnaître à Karadimas le génie d'avoir décelé le potentiel en question et de l'avoir exploité à bon escient. Enfin, comment ne pas apprécier la clarté de son écriture ?

Dimitri Karadimas nous lègue un corpus de textes et d'images extraordinaires qui atteste d'un esprit curieux de tout, inventif d'un point de vue méthodologique, généreux et courageux à la fois d'un point de vue théorique. Hélas, il nous lègue une œuvre inachevée. 